\title{
Superstructure Formation in Thermoplastic E lastomer and Oil Systems. Formation of a Novel Microcellular Porous Structure
}

\author{
Naruhiko MASHITA ${ }^{\dagger}$ and Yoshihide FUKAHORI \\ Bridgestone Corporation, Chemical and Industrial Products Division, Materials Development Department, \\ 1 Kashio-cho, Totsuka-ku, Yokohama-shi, Kanagawa-ken 244-8510, Japan
}

(Received April 3, 2002; Accepted August 21, 2002)

\begin{abstract}
A novel microcellular porous structure, the product of a small proportion of a semi-crystalline thermoplastic elastomer (TPE) and a large proportion of low molecular weight oil, is examined. The structure is formed by the phase separation of a homogeneous mixture of TPE and oil. The system exhibits a unique three-dimensional continuous polymer network consisting of interconnected spherical cells of a few tens of micrometers in diameter. The detailed phase separation process is investigated utilizing optical microscopy, SEM, and DSC. A modulated structure, apparently attributable to spinodal decomposition, is observed in the initial stage of phase separation. However, during its evolution, this structure evolves into a clear network structure of a polymer-rich phase and clusters of a spherical oil-rich phase. Time evolution of $D$ of a typical structure during the phase separation process at constant temperature is estimated to be $D \sim t^{1}$ in the initial stage and $D \sim t^{1 / 3}$ in the late stage. The character and role of differences in $M_{\mathrm{w}}$ of components in the phase separation of the TPE/oil system are discussed in relation to the results of other studies.

KEY WORDS Phase Separation / Percolation-to-Cluster Transition / Network Structure / Polymer Solution / Thermoplastic Elastomer /
\end{abstract}

The phase separation process and morphology of polymer blend systems and alloys are both attractive and important from scientific and industrial perspectives, in part because the physical and mechanical properties of such polymer systems are highly dependent on their higher-order structures. Many types of phasecontrolled polymers have been developed and put to practical use over past decades.

Despite numerous theoretical approaches, ${ }^{1-4}$ most efforts to verify experimentally the kinetics of phase separation and its progress have generally been performed by indirect methods, such as time-resolved light scattering. ${ }^{5-9}$ However, in the past five years, several studies have reported on direct observation of phase separation phenomena using laser scanning confocal microscopy, e.g., Ribbe, ${ }^{10}$ Jinnai, ${ }^{11,12}$ and Hermansson. ${ }^{13}$

Recently, the authors proposed new non-aqueous physical gels prepared with small proportions of semicrystalline thermoplastic elastomers (nearly $10 \mathrm{wt} \%$ polymer concentration) and large proportions of oils of low molecular weight. ${ }^{14,15}$ Observed directly with an optical microscope, the systems consist of threedimensional continuous polymer networks, constituted spherical cells of a few tens of micrometers in diameter. Each cell is connected to several other cells. Small openings occur at the connections between the cells: tunnels formed in the walls of the cells. This observation suggests that the oil-rich phases enclosed within the polymer cells are interconnected through the holes, and that the oil-rich phase is also continuous. We call this a "microcellular porous structure." The systems are quite stable thermally and dynamically and are currently used in industrial products as very soft nonaqueous and thermoplastic gels.

The first purpose of this study is to present detailed morphological information for the phase separation and development of a polymer blend system consisting of semi-crystalline thermoplastic elastomer and an oil of low molecular weight, using optical and scanning electron microscopy. The mechanism of phase separation and the development of the above polymer blends will be discussed by comparing them to experimental results obtained in other studies.

\section{EXPERIMENTS}

A semi-crystalline thermoplastic elastomer (TPE), poly(ethylene-propylene) rubber $\left(\mathrm{C}-\mathrm{EPR}, M_{\mathrm{w}}=\right.$ 200000 , crystallinity $=12 \%, T_{\mathrm{m}}=52^{\circ} \mathrm{C}$, trade name is EP01 produced by JSR Corp. JAPAN) was used. A non-crystalline poly(ethylene-propylene) rubber $(\mathrm{N}-$ EPR, $M_{\mathrm{w}}=220000$, trade name is EP921 produced by JSR Corp. JAPAN) was also used for comparison with semi-crystalline C-EPR. Diisodecyl adipate was used as the oil. (DIDA, $M_{\mathrm{w}}=427$, DAIHACHI-KAGAKU Corp. JAPAN.)

A homogeneous mixture was prepared by completely 
dissolving TPE in oil by stirring for one $1 \mathrm{~h}$ at $230^{\circ} \mathrm{C}$. The observation sample was prepared in the following manner: A small amount of the homogeneous solution at mixing temperature was placed on a glass plate with a cover glass, which had been heated to $230^{\circ} \mathrm{C}$ in advance. Two cooling methods were used to observe structures. First, the sample was cooled to room temperature at a constant cooling rate. Second, the sample was placed on a heat stage controlled to $90^{\circ} \mathrm{C}$ or $60^{\circ} \mathrm{C}$, then rapidly cooled to the stage temperature.

Direct observations were made with an Olympus transmission-type optical microscope with a CCD camera. Temperature conditions, including several cooling rates, were controlled by a Mettler FP82 hot stage. The process was recorded by a video-recorder, and the resulting recordings were used for structural analysis. The estimates of structure size were based on photocopies of the recorded process.

The phase diagram was determined to be the cloud point curve in the cooling process of the system. The cloud points were detected from the observation of thin samples between glass slides under the optical microscope with the hot stage. The samples were cooled at a rate $1{ }^{\circ} \mathrm{C} \mathrm{min}-1$. The onset of the decline in transmitted light intensity as phase separation arose was chosen as the cloud point temperature.

SEM observation was performed at room temperature. The sample preparation was initially soaked in acetone and left to stand for at least one night, then dried at room temperature following the replacement of oil with acetone. No compression and/or squeezing was performed during substitution or drying.

The thermal properties of the system were measured by TA Instruments DSC2920, which was purged with nitrogen gas and quenched with liquid nitrogen. The specimen (approx. $10 \mathrm{mg}$ ) was heated in a sealed hermetic aluminum pan and scanned from -100 to $150^{\circ} \mathrm{C}$, using a heat rate of $10^{\circ} \mathrm{C} \mathrm{min}^{-1}$.

\section{RESULTS AND DISCUSSION}

\section{Initiation and Evolution of the Superstructure in the System}

Structure Formation in the Cooling Process. First observed was the appearance and evolution of the superstructure. Figure 1 shows the phase diagram of the blend system of semi-crystalline EPR (C-EPR) and DIDA, a typical UCST phase diagram. The phase diagram is highly asymmetric. Figures $2 a-2 d$ show the superstructures formed at several different temperatures during the cooling process $\left(3^{\circ} \mathrm{C} \mathrm{min}^{-1}\right)$ of the sufficiently dissolved mixture of the blend system of C-EPR/DIDA (12 wt $\%$ polymer concen-

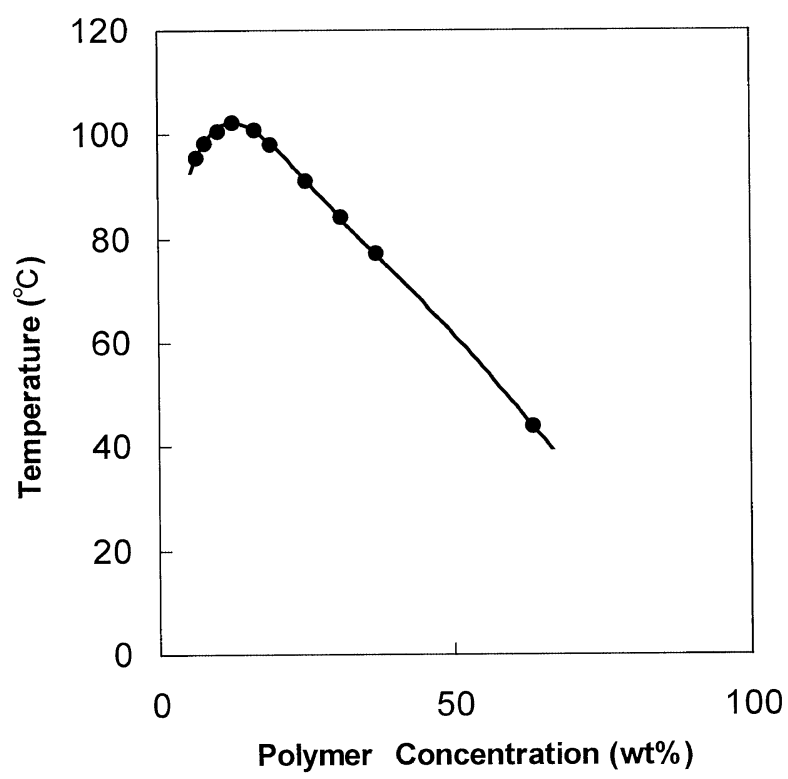

Figure 1. Phase diagram of the blend system of semicrystalline EPR (C-EPR) and DIDA.

tration). When the mixture was cooled slightly to $180^{\circ} \mathrm{C}$, an almost homogeneously dissolved state was still observed (Figure 2a). At $95^{\circ} \mathrm{C}$, a decline in transmitted light intensity was observed, apparently indicating the onset of phase separation. The structures in these figures are quite similar to the modulated structures observed in the initial stage of spinodal decomposition in polymer/polymer systems. During the development process, the polymer-rich phase appeared quite clearly as network structures, while spherical domains of the oil-rich phase appeared to be enclosed by surrounding polymer-rich networks (Figure $2 \mathrm{c}$, at $70^{\circ} \mathrm{C}$. As temperature continued to fall, the polymer network finally reached a critical network size, after which the geometrical shape of the network structure did not change (Figure 2d). In this final coarsening process of the network growth, the thickness of the polymer-rich phase appeared nearly constant, despite steadily increasing network pore size. This indicates that the phase separation and coarsening process of network formation differ from that of conventional spinodal phase separation, in which both phases increase in size self-similarly, with identical coarsening rates. Figure 3 illustrates DSC curves of the C-EPR and DIDA system ( $12 \mathrm{wt} \%$ polymer concentration) at a heating and cooling rate of $10^{\circ} \mathrm{C}$ $\mathrm{min}^{-1}$. In the cooling process, crystallization begins at nearly $35^{\circ} \mathrm{C}$ and continue to $-10^{\circ} \mathrm{C}$. In the heating process, melting occurs over the range $30-60^{\circ} \mathrm{C}$. The importance of crystallization for stabilization of the superstructure will be discussed further below. Figure 4 shows a SEM photograph of the structures (4a $12 \mathrm{wt} \%$, 4b $20 \mathrm{wt} \%$ polymer concentration). It 


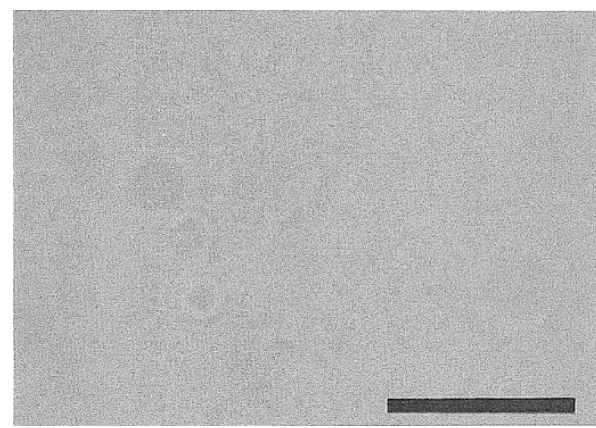

(a)

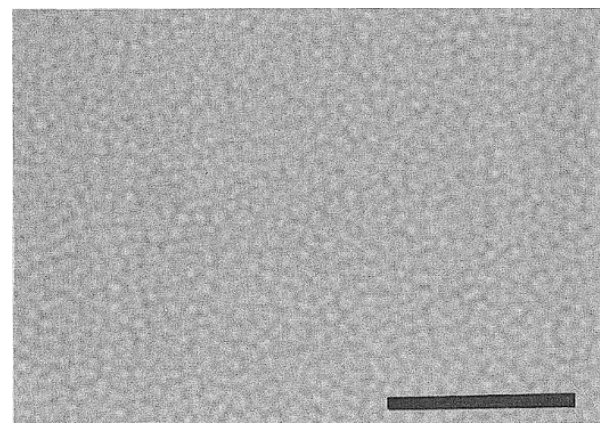

(b)

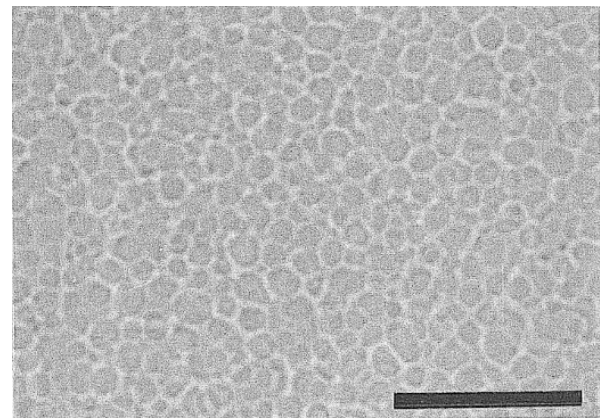

(c)

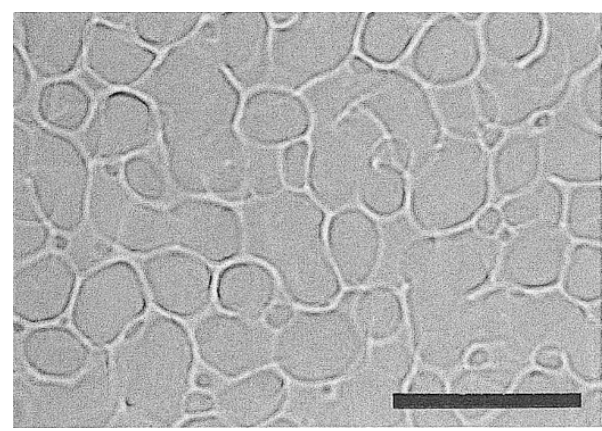

(d)

Figure 2. Phase separation during cooling process in the $\mathrm{C}$ EPR/DIDA system (12 $\mathrm{wt} \%$ polymer concentration): $180^{\circ} \mathrm{C}(\mathrm{a})$, $95^{\circ} \mathrm{C}(\mathrm{b}), 70^{\circ} \mathrm{C}(\mathrm{c})$, and $20^{\circ} \mathrm{C}(\mathrm{d})$. Bar $=100 \mu \mathrm{m}$.

was separately ascertained that the network structure is relatively unaffected by removing the oil-rich phase in SEM observation. The SEM photograph shows the appearance of interconnected cells of the polymer-rich phase. A portion of the edge of a cell was observed as a network structure (Figures 2c, 2d, and Figure 4a). Where the cells are connected, more than one hole

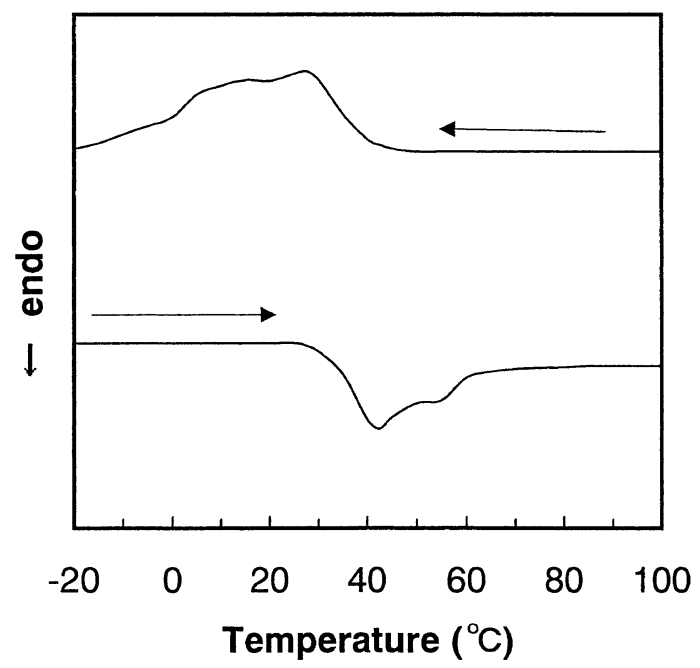

Figure 3. DSC curves of the C-EPR/DIDA system (12 wt\% polymer concentration) with heating and cooling rate of $10{ }^{\circ} \mathrm{C}$ $\min ^{-1}$.

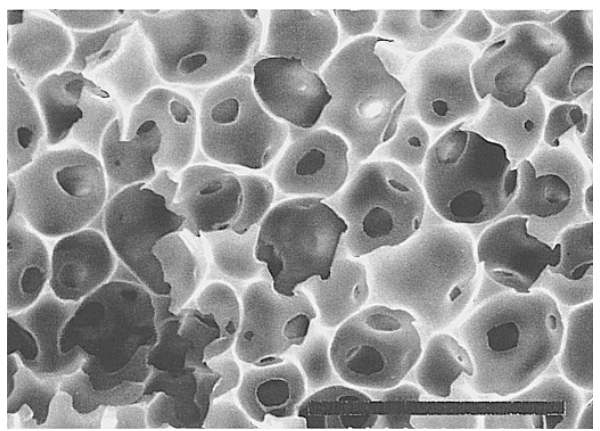

(a)

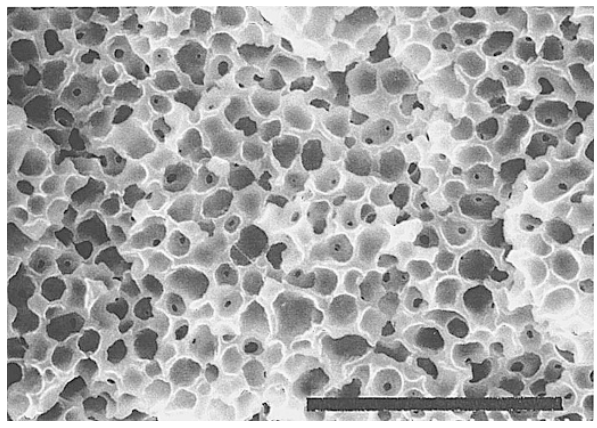

(b)

Figure 4. Scanning electron micrographs of the C-EPR/DIDA system: $12 \mathrm{wt} \%$ polymer concentration (a), $20 \mathrm{wt} \%$ polymer concentration (b). Bar $=100 \mu \mathrm{m}$.

is formed, through which the oil-rich phase retained within the cells are interconnected and kept continuous. This photograph shows that the polymer blend system has a microcellular porous structure consisting of polymer-rich and oil-rich phases.

Structure Formation at a Constant Temperature. Figure 5 (optical microphotographs) shows phase structure formation when the system is quenched and kept at constant temperature of $60^{\circ} \mathrm{C}$. The system was homogeneously mixed at $230^{\circ} \mathrm{C}$, then quenched to $60^{\circ} \mathrm{C}$. 


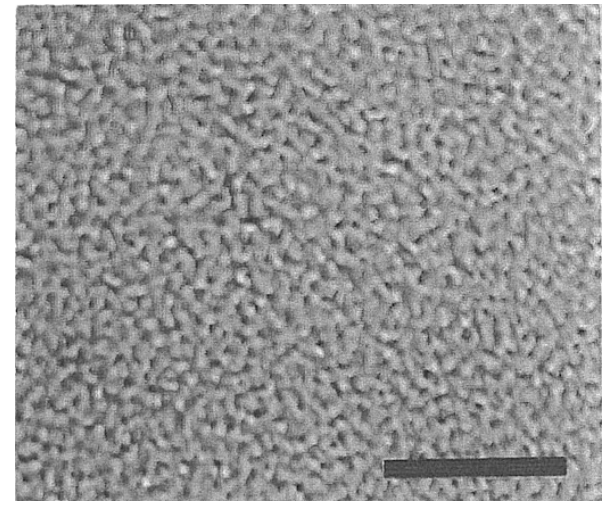

(a)

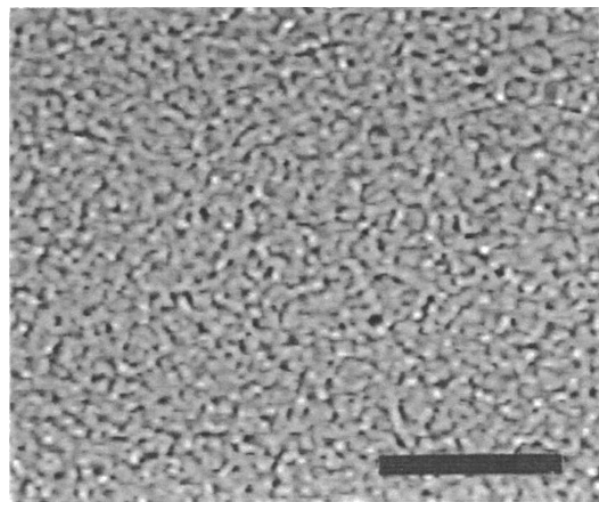

(b)

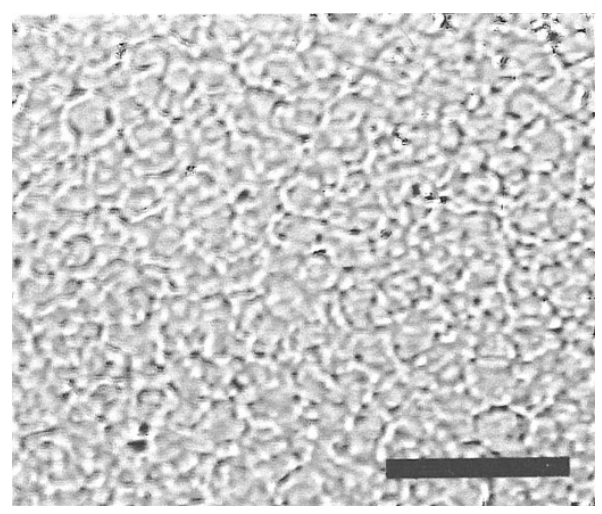

(c)

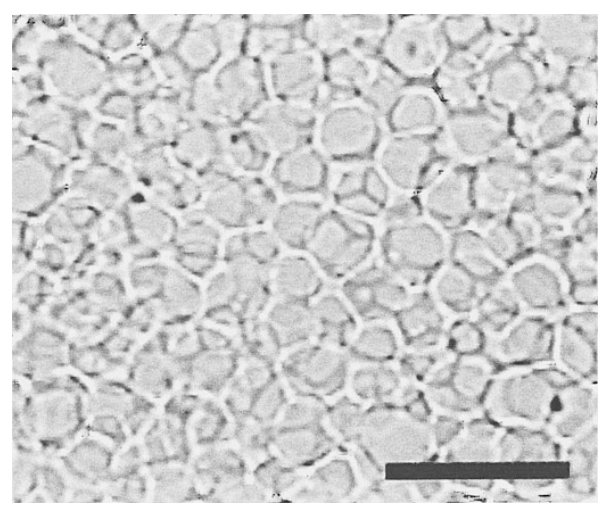

(d)

Figure 5. Phase separation at $60^{\circ} \mathrm{C}$ in the C-EPR/DIDA system (12 $\mathrm{wt} \%$ polymer concentration): $0.5 \mathrm{~min}$ after quenching (a), $1.0 \mathrm{~min}(\mathrm{~b}), 1.5 \mathrm{~min}(\mathrm{c})$, and $10 \mathrm{~min}(\mathrm{~d}) . \mathrm{Bar}=100 \mu \mathrm{m}$.

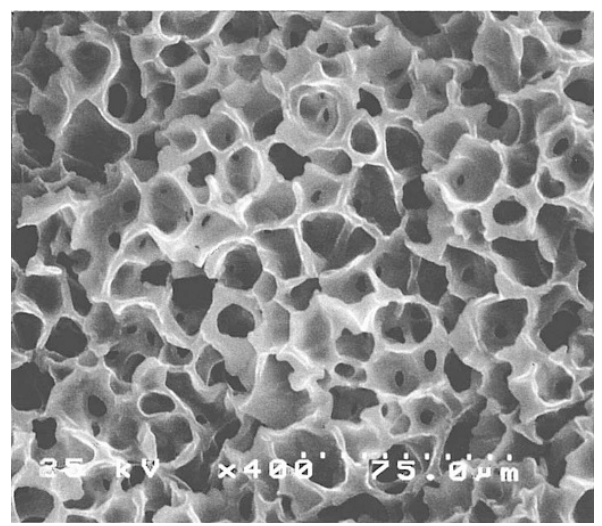

Figure 6. Scanning electron micrograph of the C-EPR/DIDA system (12 $\mathrm{wt} \%$ polymer concentration).

The structures observed after $30 \mathrm{sec}$ (Figure 5a) and $60 \mathrm{sec}$ (Figure 5b) are likely the modulated structures generally observed in the initial stage of spinodal decomposition. However, after 1.0 min of quenching, the polymer-rich phase forms a network everywhere within the field of view of the microscope. After $1.5 \mathrm{~min}$, the network structure forms over virtually the entire area (Figure 5c). The network structure continues to become significantly more clearly defined, with the diameter of the network expanding as coalescence progressed (Figure 5d). Figure 6 shows a SEM photograph of the structure observed in Figure 5d. The network structure seen in Figure 5d is taken to represent spherical cellar connective tissue. The evolution of the phase structure was observed by the same procedure when the homogeneous mixture was quenched to $90^{\circ} \mathrm{C}$ and held at constant temperature. These situations in structure formation are basically identical to the case observed in the cooling process (Figure 2).

The typical diameter $D$, which shows the size of the structure as a function of time $t$, is plotted in Figure 7. The length (width) in the minor axis of the oil-rich phase was measured for a typical size $D$ in the structure. In the period following the appearance of the network structure, the diameter of the oil-rich phase, which encompasses a typical area in the polymer-rich phase, is plotted as $D$. Typical cases of the size $D$ are illustrated in Figure 7. Since $t=0$ was defined as the time at which the system was quenched to constant temperature, about $5 \mathrm{sec}$ from the beginning of quenching might be required for the system temperature to settle. (This is disregarded in the plots.) From Figure 7, the gradient of the line clearly changes when $D$ reaches 10 to $15 \mu \mathrm{m}$. In Figure 7, the dotted line for $D \propto t^{1}$ for the initial time region and the solid line for $D \propto t^{1 / 3}$ for the late phase separation region are superimposed. It can be seen that in the case of quenching to $60^{\circ} \mathrm{C}$, at $t_{\mathrm{c}}=$ about $1.5 \mathrm{~min}, D_{\mathrm{c}}$ of about $13 \mu \mathrm{m}$ is a critical value, and that the slope on the short time side is 1 and that on 


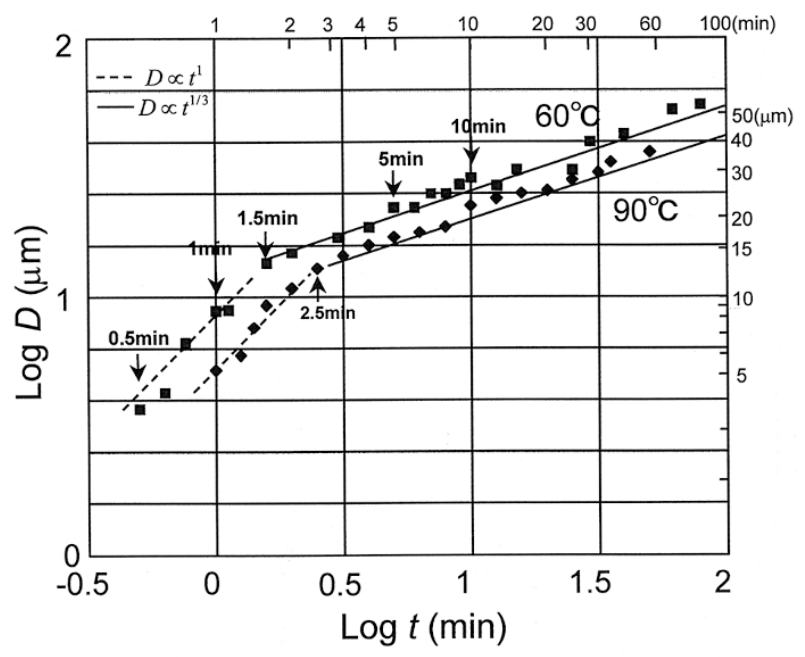

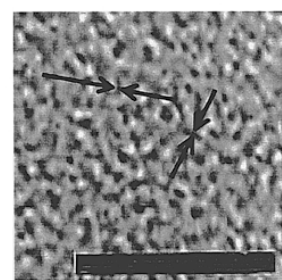

(a)

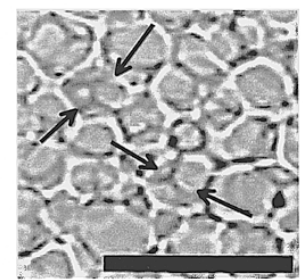

(b)
Figure 7. Time-evolution of oil-rich phase as a function of time in the C-EPR/DIDA system ( $12 \mathrm{wt} \%$ polymer concentration) at $60{ }^{\circ} \mathrm{C}$ and $90^{\circ} \mathrm{C}$. Illustrations of measurement of typical size $D$, about $0.5 \mathrm{~min}$ after quench at $60^{\circ} \mathrm{C}(\mathrm{a})$, and about $10 \mathrm{~min}$ after quench at $60^{\circ} \mathrm{C}(\mathrm{b})$. Bar $=100 \mu \mathrm{m}$

the longer side is $1 / 3$. The results measured at $90^{\circ} \mathrm{C}$ are fundamentally the same as the results at $60^{\circ} \mathrm{C}$, although the critical value $\left(t_{\mathrm{c}}=2.5 \mathrm{~min}\right)$ shifts slightly to a longer time. The critical time $t_{\mathrm{c}}$ in Figure 7 almost corresponds to time $t_{\mathrm{T}}(1.5 \mathrm{~min})$ of the change from the modulated structure to the network structure shown in Figure $5 \mathrm{c}$. This result was also confirmed by the results of $90^{\circ} \mathrm{C}$.

Alteration of Time-Evolution of Phase Separation. In the figure for the previous section, which showed the time dependence of diameter $D$ of a typical structure, the relation $D \propto t^{1}$ is observed in the initial stage of phase separation, while $D \propto t^{1 / 3}$ is observed in the late stage. The familiar theories presented to date assume that phase separation develops through a single mechanism throughout the entire process. The potential alteration of the time-evolution of phase separation has not yet been proposed.

In the $1990 \mathrm{~s}$, Hashimoto et al. ${ }^{16}$ investigated the spinodal decomposition of off-critical polymer/polymer mixtures by means of time-resolved light scattering, finding that time changes of $I_{\mathrm{m}}$, the maximum scattering intensity, and $q_{\mathrm{m}}$, the magnitude of the scattering vector at which the intensity reaches its highest value, are spontaneously pinned during development. They conjectured that the spontaneous pinning phe- nomenon is associated with a dynamical percolationto-cluster (PC) transition wherein a bicontinuous, percolating structure originating with spinodal decomposition evolves into a cluster of droplets of a minority component. The study also proposed that such pinning might originate in a kinetic barrier that suppresses the diffusion of polymer-molecules. After the initial report of Hashimoto, Gronski et al. ${ }^{17}$ studied the PC transition by optical micrographs, as well as light scattering. They observed that the morphology evolves from a percolating structure to clusters of a globular shape of a minority component around the time at which the PC transition is detected by light scattering. Thereafter, the isolated small particles coalesce into large clusters. $\mathrm{Crist}^{18}$ has asserted that the PC transition is the crossover between two kinetic regimes associated with morphology changes, and that the "pinning" observed therefore represents the induction period, a function of the molecular weight of the polymer and the size of the isolated domain, required for classical coarsening to become noticeable after PC transition. He accordingly believed that the growth laws such as $D \propto t^{1 / 3}$ would once again apply after an estimated induction period of approximately ten times the duration and a short crossover period, which leads $D \propto t^{1 / 3}$ just after $D \propto t$, proposing this as a distinct possibility in the case of the low molecular weight systems. This remarkable phenomenon has been studied both through experimentally and numerical simulations. Quite recently, Hashimoto et al. ${ }^{19}$ reported that the growth of $q_{\mathrm{m}}$ of a polymer/polymer system, which had apparently halted around the PC transition, exhibits $q_{\mathrm{m}} \propto t^{-0.33}$ over extended periods.

Thus, in recent years, many studies have investigated experimentally, and in great detail, phenomena related to morphology, the phase separation mechanism, and the time-evolution of phase growth changes during development.

We now compare the results of the TPE/oil system to the preceding studies. First, with respect to similarities between results, the time-evolution of phase is proportional to time at the initial stage, after which it begins to slow. Furthermore, both cases exhibit morphological changes; that is, changes from modulated structure into a phase structure having clusters of spherical domains. On the other hand, with respect to differences between polymer/polymer systems and TPE/oil systems, to our knowledge, the systems discussed in all reports concerning the PC transition have involved off-critical mixtures of polymer/polymer systems although this particular system involved macromolecules/small molecules. From the morphological perspective, the minority component certainly creates isolated spherical domains in those off-critical polymer/polymer mixtures; however, 

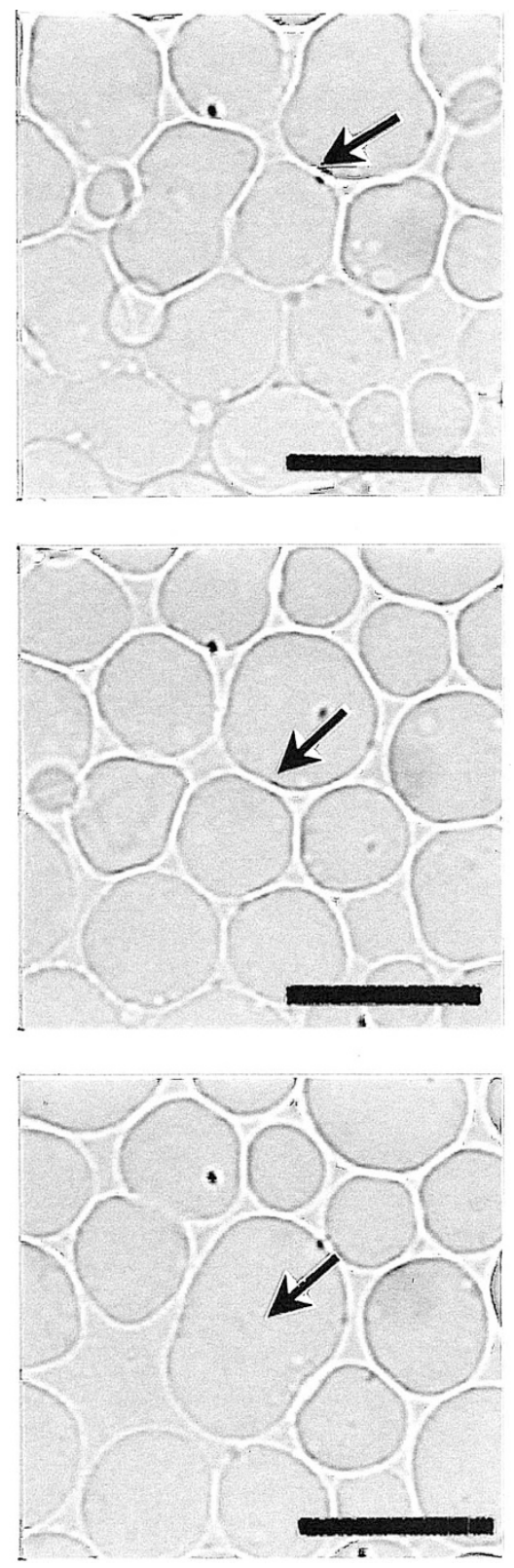

Figure 8. Coalescence of spherical oil-rich phase in the late stage (around $70 \mathrm{~min}$ after phase separation occurs) of the phase separation of the C-EPR/DIDA system ( $12 \mathrm{wt} \%$ polymer concentration) at $60^{\circ} \mathrm{C}$. The arrow points a coalescing part. Bar $=100 \mu \mathrm{m}$.

in the TPE/oil system the modulated structure evolves into a clear network structure of a minority TPE-rich phase and clusters of spherical domains of an oil-rich phase, the major component, to be enclosed by a surrounding polymer-rich network.

The percolating, bicontinuous phase structure originating in spinodal decomposition is generally said to develop rapidly by hydrodynamic coarsening, in cases where the characteristic length of the structure develops linearly with time. ${ }^{17}$ In the TPE/oil system, hydrodynamic flow may be the major mechanism of structurecoarsening in the initial stage at which $D \propto t$.

Now we consider the late stage, where $D \propto t^{1 / 3}$. Figure 8 shows the optical micrographs of phase separation in a very late stage at $60^{\circ} \mathrm{C}$. The coarsening process is easily observed, since it proceeds slowly at around the 70 min after the onset of phase separation. The coalescence of spherical oil-rich domain as the major process of coarsening is clearly observed. Apparently a driving force is to reduce the interfacial energy between the polymer-rich phase and the oil-rich phase. In passing, when the process is viewed from the opposite phase, the movement of the polymer-rich phase in observation of such coalescence process of oil-rich domain can be interpreted as the diffusing and moving process of network phase of polymer-rich phase surrounding the spherical oil-rich domains, in which case such movement is the governing process. That is, in the later stage of the TPE/oil system, the major process that controls coarsening believed to be the diffusion of the polymernetwork-phase: thus, the estimated time-evolution of the size of oil-rich phase slows to $D \propto t^{1 / 3}$.

What explains this alteration? More experimental and theoretical studies will be required to provide a reasonable answer. However, one potential cause may involve the difference in mobility arising from the difference in the molecular weight between the polymer and the oil of low molecular weight. As reported in our previous paper, ${ }^{15}$ in the case of semi-crystalline hydrogenated polybutadiene TPE and polyisobutylene oil systems, when the molecular weight of the oil is less than 1000 , the polymer's network structures are clearly visible. However, in the case of a higher molecular weight oil, $M_{\mathrm{w}}=1400$, such homogeneous regular network structures are no longer observed. In particular, in the case of $M_{\mathrm{w}}=2900$ polyisobutylene oil, no network structures at all are formed in the system. Thus, it appears that the molecular weight of oil is less than nearly one thousand, much lower than that of TPE, to form a clear network structure in this polymer/oil system.

Comparison with Other Concept. A report by Tanaka ${ }^{20}$ presents an example of a network-like structure in a system of poly(vinyl-1-methyl ether) and water, although the network-like structure appeared only in the middle stage of phase separation and finally changed to droplets. Tanaka ${ }^{21}$ explained the phenomenon in light of the importance of viscoelastic effects on phase separation and proposed a new concept of dynamic asymmetry.

Araki and Tanaka ${ }^{22}$ recently further investigated the topological change of domain morphology during phase separation by means of curvature analysis, using computer simulation. They state that network-like bicontinuous pattern of saddle-like structure is characterized by negative $K$ and negative $H$, where $K$ and $H$ refer to Gaussian and mean curvature, respectively.

Curvature analysis is becoming increasing useful for 
structure analysis and classification for phase separation structure, ${ }^{23,24}$ since three-dimensional observation by laser scanning confocal microscopy and numerical simulation can be applied to the field of morphological research in condensed matter. We wish to introduce such perspective for the morphology of the TPE/oil system. Figure 4 and Figure 6, SEM pictures of the TPE and oil system, demonstrate that the TPE and oil system basically consists of a spherical cell structure, although the cells are interconnected. Both the Gaussian $K$ and mean $H$ curvature of spherical surface are positive, as seen from the inside of the sphere, as is well-known from differential geometry. ${ }^{25}$ Thus, the curvature of the TPE/oil system is generally positive, and the morphology of the TPE/oil system can be placed in a category different from Tanaka's system, although further morphological study of the TPE/oil system is required.

\section{Fixing and Stabilization of the Superstructure}

Structure Formation and Stabilization of the Superstructure Produced in Non-crystalline TPE and Oil System. To analyze the effects of the crystallinity of polymer in the formation and stabilization of the network structure, a mixture of non-crystalline EPR (N-EPR)/DIDA (12 $\mathrm{wt} \%$ polymer concentration) was prepared and cooled in the same manner as the CEPR/DIDA system. Figures 9a-9d show the formation and collapse of the superstructures occurring during the cooling process from $230^{\circ} \mathrm{C}$ to room temperature in N-EPR and DIDA system. Phase separation occurs when the homogeneously dissolved mixture is cooled to $55^{\circ} \mathrm{C}$ (Figure 9a). Although this occurs at a temperature much lower than in crystalline EPR, the structure increased in size and evolved to network structure similar to those observed in crystalline EPR (Figure 2). The network increased in size slightly during further declines in temperature to room temperature, but remained significantly below $1 / 3-1 / 5$, of that in crystalline EPR (Figure 9b). After $30 \mathrm{~min}$ at room temperature, the network structures thus developed began to collapse partially (Figure 9c), becoming droplets and dispersing in the oil-rich matrix after $2 \mathrm{~h}$ (Figure 9d).

These phenomena indicate that although the superstructure arises and evolves almost identically in the crystalline and non-crystalline TPE and oil systems, the structure is fixed and stabilized only in the former, collapsing and separating again into distinct polymer and oil phases in the latter. This occurs as a result of the force balance between the elastic force of the semi-crystalline polymer-rich network and the interfacial tension of the oil-rich droplets. If the elastic force overcomes the interfacial force, the coalescence of droplets may be prevented and consequently, the

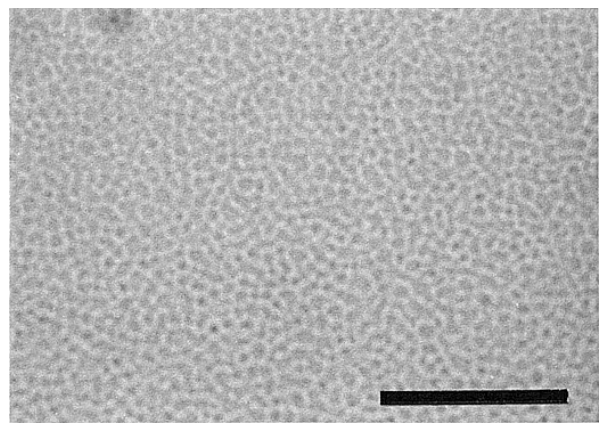

(a)

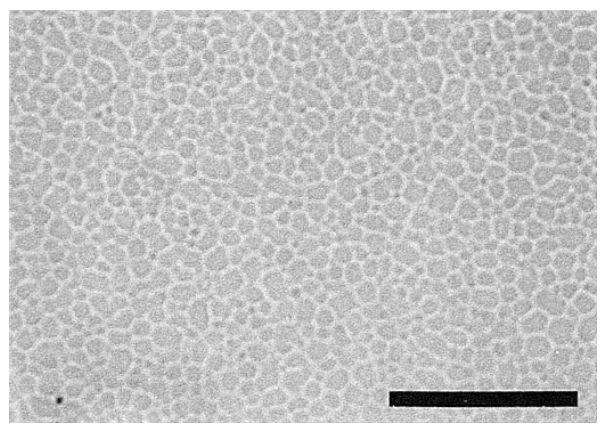

(b)

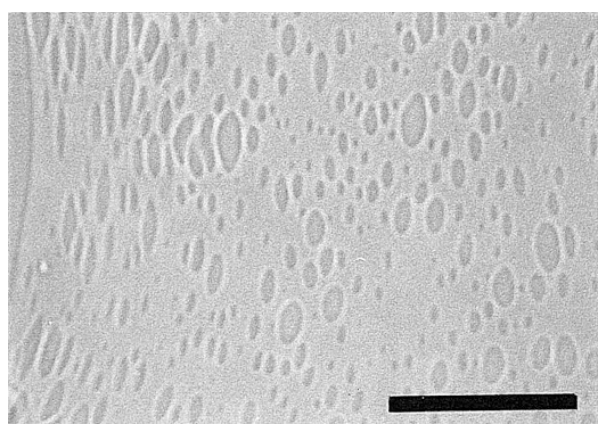

(c)

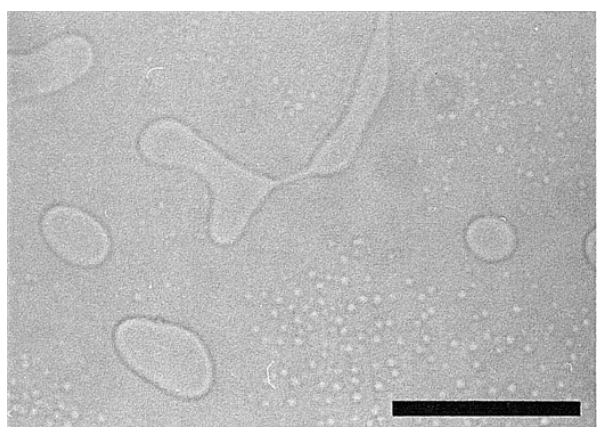

(d)

Figure 9. Phase separation during cooling process in the $\mathrm{N}$ EPR/DIDA (12 $\mathrm{wt} \%$ polymer concentration) system: $55^{\circ} \mathrm{C}$ (a),

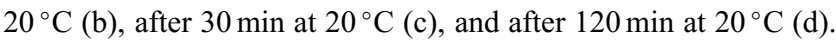
Bar $=100 \mu \mathrm{m}$.

coarsening of the network may arrest, something that occurs in the semi-crystalline TPE/oil system.

\section{SUMMARY}

We introduced a unique network structure forma- 
tion in a system consisting of a small portion of semicrystalline TPE and a large portion of oil. The phase separation process began as likely spinodal decomposition, but the modulated structure evolved during development into a clear microcellular porous structure of a polymer-rich phase and clusters of spherical oilrich phase. In this report, we focused time-evolution of scale $(D)$ of the oil-rich phase during the phase separation process at constant temperature. It is estimated that $D \sim t^{1}$ in the initial stage and $D \sim t^{1 / 3}$ in the late stage. We discussed that the character and role of asymmetry of components in the phase separation of the TPE/oil system.

Acknowledgments. N. M. thanks Dr. Xiaorong Wang of Bridgestone/Firestone Research, Dr. Hideaki Yokoyama of National Institute of Advanced Industrial Science and Technology (AIST), Mr. Takahiro Matsuse and Dr. Katsuhiko Tsunoda of Bridgestone Corp. for valuable discussion. The authors also acknowledge the technical support and discussion of Mr. Yuichiro Wakana and Mr. Yuji Kitamura of Bridgestone Corp. We gratefully acknowledge the member of MNCSMicro Network Controlled Structure-project in Bridgestone group worldwide.

\section{REFERENCES}

1. I. M. Lifshiz and V. V. Slyozov, J. Phys. Chem. Solids, 19, 35 (1961).

2. J. W. Cahn, J. Phys. Chem., 41, 93 (1965).

3. K. Binder and D. Stauffer, Phys. Rev. Lett., 33, 1006 (1974).

4. E. D. Siggia, Phys. Rev. A, 20, 595 (1979).

5. T. Hashimoto, J. Kumaki, and H. Kawai, Macromolecules, 16, 541 (1983).
6. H. L. Snyder and P. J. Meakin, J. Chem. Phys., 79, 5588 (1983).

7. N. Kuwahara, H. Sato, and K. Kuboto, J. Chem. Phys. Commun., 97, 5905 (1992).

8. F. J. Bates and P. J. Wilzius, Chem. Phys., 92, 3208 (1989).

9. T. Izumitani and T. Hashimoto, J. Chem. Phys., 83, 3694 (1985).

10. A. E. Ribbe and T. Hashimoto, Macromolecules, 30, 3999 (1997).

11. H. Jinnai, T. Koga, Y. Nishikawa, T. Hashimoto, and S. T. Hyde, Phys. Rev. Lett., 78, 2248 (1997).

12. H. Jinnai, Y. Nishikawa, H. Morimoto, T. Koga, and T. Hashimoto, Langumuir, 16, 4380 (2000).

13. L. Niklas, A. Altskar, and A.-M. Hermansson, Macromolecules, 34, 8117 (2001).

14. Y. Fukahori and N. Mashita, Polym. Prepr. Jpn., 44, 1738 (1995).

Y. Fukahori and N. Mashita, Polym. Prepr. Jpn., 44, 1740 (1995).

15. Y. Fukahori and N. Mashita, Polym. Adv. Technol., 11, 472 (2000).

16. T. Hashimoto, M. Takenaka, and T. Izumitani, J. Chem. Phys., 97, 679 (1992).

17. J. Lauger, R. Lay, and W. Gronski, J. Chem. Phys., 101, 7181 (1994).

18. B. Crist, Macromolecules, 29, 7276 (1996).

19. H. Takeno, M. Iwata, M. Takenaka, and T. Hashimoto, Macromolecules, 33, 9657 (2000).

20. H. Tanaka, J. Chem. Phys., 100, 5323 (1994).

21. H. Tanaka, J. Phys.: Condens. Matter, 12, 207 (2000).

22. T. Araki and H. Tanaka, Macromolecules, 34, 1953 (2001).

23. H. Jinnai, T. Koga, Y. Nishikawa, T. Hashimoto, and T. S. Hyde, Phys. Rev. Let., 78, 2248 (1997).

24. H. Jinnai, Y. Nishikawa, H. Morimoto, T. Koga, and T. Hashimoto, Langmuir, 16, 4380 (2000).

25. S. Kobayashi, "Differential Geometry of Curves and Surfaces", Shokabo, Tokyo, 1995, chapt. 2. 